\title{
Hybrid Quantum Information Processing
}

\author{
Ulrik L. Andersen,, , Jonas S. Neergaard-Nielsen, ${ }^{1}$ Peter van Loock, ${ }^{2}$ and Akira Furusawa ${ }^{3}$ \\ ${ }^{1}$ Department of Physics, Technical University of Denmark, Fysikvej, 2800 Kongens Lyngby, Denmark \\ ${ }^{2}$ Institute of Physics, Johannes-Gutenberg Universität Mainz, Staudingerweg 7, 55128 Mainz, Germany \\ ${ }^{3}$ Department of Applied Physics, School of Engineering, \\ The University of Tokyo, 7-3-1 Hongo, Bunkyo-ku, Tokyo 113-8656, Japan
}

The development of quantum information processing has traditionally followed two separate and not immediately connected lines of study. The main line has focused on the implementation of quantum bit (qubit) based protocols whereas the other line has been devoted to implementations based on high-dimensional Gaussian states (such as coherent and squeezed states). The separation has been driven by the experimental difficulty in interconnecting the standard technologies of the two lines. However, in recent years, there has been a significant experimental progress in refining and connecting the technologies of the two fields which has resulted in the development and experimental realization of numerous new hybrid protocols. In this Review, we summarize these recent efforts on hybridizing the two types of schemes based on discrete and continuous variables.

\section{INTRODUCTION}

By harnessing the quantum effects of superposition and entanglement, revolutionary new methods of communication and computation can be realized 11. A prominent example is the possibility of generating a secret key between two or more parties in a communication network leading to unconditionally secure communication known as quantum cryptography. Another striking example, and perhaps the most intriguing one, is the realization of a quantum computer which allows for exponentially faster computation of certain tasks. Although the experimental progress in controlling quantum states of various microscopic quantum systems has exploded in recent years, the implementation of a fully fault-tolerant and scalable quantum computer is still a major challenge.

Spurred by the grand vision of constructing a quantum computer, numerous physical platforms are being intensely explored worldwide. These include light, ions, atoms, solid state, cavity quantum electrodynamics, superconducting systems and nuclear magnetic resonance 22. However, irrespective of the physical system, quantum information processing (QIP) comes in two different forms depending on which degree of free-

\footnotetext{
${ }^{*}$ Corresponding author ulrik.andersen@fysik.dtu.dk
}

dom, or observable, is being used for describing the involved quantum states. If this observable is of discrete nature (that is, its eigenvalues are discretized), one often refers to discrete-variable (DV) QIP (Box 1), and if the observable has a continuum of eigenvalues, one talks about continuous-variable (CV) QIP (Box 2). An analog can be drawn to classical information processing where the two forms exist in the realm of digital and analog information processing.

In recent years, numerous research groups have worked on bridging the two islands with the aim of realizing protocols that overcome the intrinsic limitations of the individual DV and CV schemes. The integration of DV and CV technologies in unified hybrid optical systems has thus resulted in a series of new proposals and groundbreaking experiments realizing long-standing goals 3. The aim of this Review is to present the recent progress on combining the CV and DV methods for applications in QIP. A major research effort has been devoted to the generation of highly nonclassical quantum states using hybrid technologies. These efforts will be reviewed in section III In section III] we will review QIP including quantum teleportation, quantum repeaters and quantum computing based on hybrid schemes, and finally, in section IV, we conclude the review with an outlook.

\section{GENERATION OF NON-GAUSSIAN STATES}

There are two classes of pure quantum states that play a pivotal role in quantum information processing: Gaussian states and non-Gaussian states, referring to the statistics of the state's wavefunction or Wigner function (Box 3). The Gaussian states - e.g. coherent and squeezed states - are often referred to as CV states. They are relatively easy to produce and manipulate using standard CV technology such as lasers, parametric amplifiers (or squeezers), beam splitters and homodyne detectors. This technology enables a linear transformation of continuous quantum quadratures thereby mapping a Gaussian state onto another Gaussian state [15 17. Such transformations have been mastered in the optical regime for more than two decades, but in recent years it has been also extended to the microwave regime: Using superconducting degenerate [18 and non-degenerate [19, 20] parametric amplifiers, microwave squeezed and CV entangled states have been generated and characterized with homodyne detection for state tomography [21]. In addition to the generation of squeezing of the field quadratures, there 
Box 1 Discrete-variable quantum information processing

A single photon can carry information in different degrees of freedom such as its polarization, its time of arrival and spatial mode whereas an electron can carry information in its spins. Binary digits can thus be represented by orthogonal eigenstates of a single photon or a single electron. In quantum information, the information is not solely comprised by the eigenstates, but can be encoded as superpositions of the eigenstates:

$$
|\psi\rangle=c_{0}|0\rangle+c_{1}|1\rangle
$$

which is known as the qubit. The information of this quantum state is given by the complex amplitudes $c_{0}$ and $c_{1}$, and it can be visualized on the Bloch sphere. In this system the computational basis set $\{|0\rangle,|1\rangle\}$ is discrete and limited to two. The measurement of this qubit is described by a two-component projector such that in each measurement the number of outcomes (eigenvalues) is limited to two. An example is a Stokes parameters measurement (consisting of waveplates, a polarizing beam splitter and two photon counters) or a Stern-Gerlach apparatus both of which ideally projects along any orthogonal basis. A universal two-component projector can be used to implement a measurement induced non-linearity and it can be used to fully characterize a state in the two-dimensional Hilbert space.

In order to implement universal quantum computation and perform a complete set of quantum communication tasks, a finite set of gates comprising single qubit and two-qubit operations must be implemented. One example of a complete set is $\left\{\hat{U}_{H}, \hat{U}_{P G}, \hat{U}_{C N O T}\right\}$ where $\hat{U}_{H}$ and $\hat{U}_{P G}$ are the single qubit Hadamard and rotation gates and $\hat{U}_{C N O T}$ is the two qubit controlled NOT gate. Deterministic single qubit gates can be readily performed with simple linear optics but the deterministic two-mode gate requires the introduction of a very large non-linearity. These hard interactions can however be by-passed using probabilistic measurement induced operations as suggested in ref. 4] but the required overhead is massive [5].

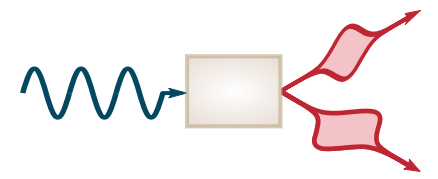

photon pair generation in lowgain parametric down-conversion

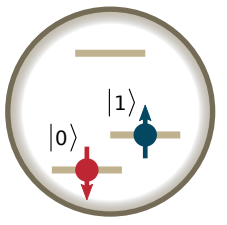

electron spin

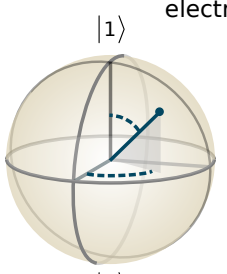

$|0\rangle$

qubit Bloch sphere

\begin{abstract}
spin
\end{abstract}

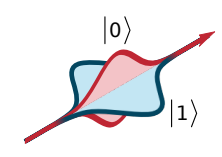

polarization qubit

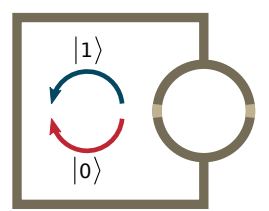

flux qubit

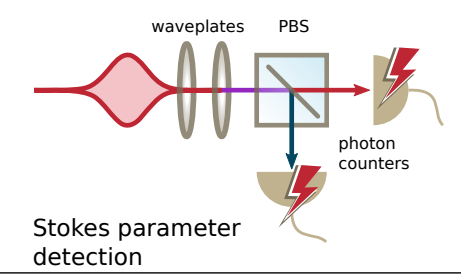

Box 2 Continuous-variable quantum information processing

As an alternative to the standard finite-level encoding, one might use a continuous basis $\{|x\rangle\}$ for the encoding, examples being the amplitude and phase quadratures of a field mode, the postion and momentum of a mechanical oscillator and the spin variables of an atomic ensemble. An arbitrary quantum state in this basis is

$$
|\psi\rangle=\int \psi(x)|x\rangle d x
$$

where the information is now contained in the wavefunction $\psi(x)$ rather than in discrete numbers as for the twolevel system (see Box 1). If $\psi(x)$ is Gaussian, the state is coined Gaussian which is the case for the coherent state, the squeezed state and the CV entangled state (also known as the two-mode squeezed state). In optics, the position eigenstates correspond to a quadrature eigenstate which physically represents an infinitely squeezed state displaced to the quadrature value $x$. A measurement of the basis states is done with a continuous projector which physically can be carried out with high efficiency using a homodyne detector. The outcomes of such measurements, that is, the eigenvalues, are now continuous numbers. Using such a homodyne projector, it is possible to perform complete tomography of any quantum state of light.

A universal set of gates for continuous-variable computation has been defined and they can basically be categorized in two types of transformations; Gaussian and nonGaussian transformations: $\left\{\hat{F}, \hat{Z}, \hat{U}_{S U M}, \hat{U}_{P G}\right\}$ including the single mode Gaussian gates (Fourier transform and displacement), the g Gaussian SUM gate and the twomode non-Gaussian cubic phase gate. The Gaussian transformations are standard in a CV laboratory and sufficient for a large range of protocols 6 9]. However, universality is only attained by the technically challenging non-Gaussian transformations [10 14].

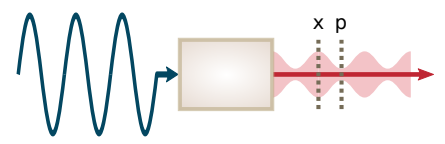

squeezed light generation in highgain parametric down-conversion
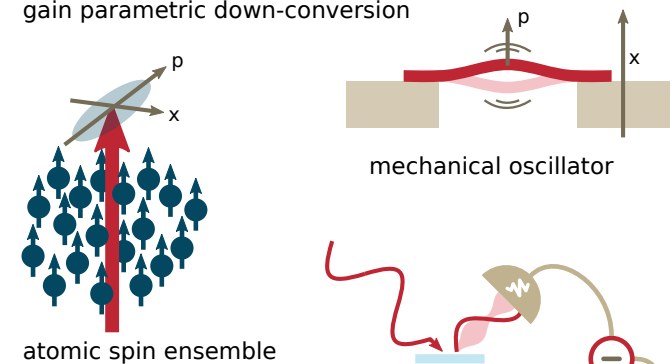

mechanical oscillator

atomic spin ensemble

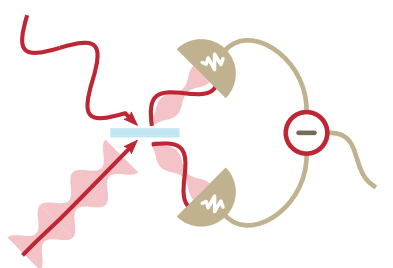

homodyne detector

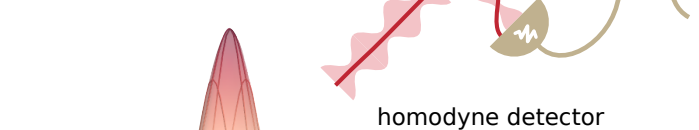

Wigner function 
Box 3 The Wigner function

The quantum state of an optical field can be represented as a distribution $W(x, p)$ over the continuous-variable phase space. This Wigner function has a one-to-one correspondence with the density matrix, and therefore contains all information about the state. It is very similar to a classical probability distribution, but it can attain negative values. This property, closely related to Heisenberg's uncertainty principle, provides a straight-forward criterium for the non-classicality of a quantum state. Among pure states, only the Gaussian ones do not exhibit negativity of their Wigner functions. But negativity is fragile, so it can quickly vanish from a non-Gaussian state that has lost its purity. Observation of non-Gaussian states with negative Wigner functions therefore require high efficiencies throughout the system. The Wigner function can be reconstructed by the method of homodyne tomography, where multiple identical copies of the quantum state are prepared and measured on a homodyne detector with different settings of the phase of the local oscillator. Alternatively, it can reconstructed via displacement controlled parity measurement.

has also been some recent demonstrations on squeezing the CV collective spin observables of an atomic ensemble [22] and similar proposals exist for solid state materials [23, 24]. Within the last decade, there has been massive interest in generating and manipulating the position and momentum CVs of mechanical oscillators. This has lead to numerous proposals on generating mechanically squeezed and entangled states exploiting the Gaussian coupling between a field mode and the mechanics 25. This has very recently been accomplished in a superconducting system in which entanglement between a microwave field and a mechanical oscillator was created 26].

To produce pure non-Gaussian states, and in general an arbitrary quantum state, the standard CV toolbox consisting of linear Gaussian transformation and homodyne detection is insufficient. It is however possible to enter the non-Gaussian regime by hybridizing DV and CV technology. There are basically two approaches to the formation of non-Gaussian states of an oscillator: 1) By enabling a strong, deterministic coupling to a finitelevel (discretized) matter system or 2) by a probabilistic measurement-induced interaction using a finite-level discretized energy detector (photon counter).

Deterministic generation of non-Gaussian states using two-level matter systems. The interaction between a CV oscillator and a DV two-level system can be described by the Jaynes-Cummings interaction. The simplest non-Gaussian state to produce using such an interaction is the single photon state; each time the two-level system is excited, it will decay and emit a single flying photon into a travelling field mode. If a single field mode is strongly coupled to the two-level system - usually enabled by placing the systems inside a high Q cavity - the photon will be harvested by that mode with large probability. This is known as the Purcell effect. It has been demonstrated in a number of experiments [27, but a complete state characterization via Wigner function reconstruction has been realized only in a few experiments, mainly in the microwave regime [28 31], but recently also in the optical regime with atomic ensembles [32, 33.

In the microwave domain, the coupling strength can be enormously high by employing a superconducting phase qubit inside a microstrip cavity, and moreover, the coupling can be controlled by detuning the cavity in and out of resonance with the field 34. Using such a strong and controlled coupling, higher order Fock states 35 as well as Fock state superpositions of several photons 36. have been deterministically generated and characterized by quantum state tomography on a chip in a cryogenic environment, see Fig. 1 p. Similar multi-photon Fock states have been generated via quantum non-demolition measurement of a stationary microwave field employing the strong coupling and subsequent measurements of flying Rydberg atoms [28, see Fig. 17.

Another non-Gaussian state of the harmonic oscillator that has attracted significant interest is the so-called "Schrödinger cat state". It is defined as a superposition of macroscopic states. Examples are the superpositions of coherent states with opposite phase, $|\alpha\rangle+e^{i \phi}|-\alpha\rangle$ 37. where $\alpha$ is the coherent state amplitude and $\phi$ is a phase. The name is a reference to Schrödinger's famous Gedankenexperiment [38]: The coherent states are seen as macroscopically distinct states, "alive" and "dead," and the cat state is in a superposition of these. Such states have been deterministically realized in the motional state of a trapped ion [39] and in the microwave regimes by entangling a standing $\mathrm{CV}$ microwave field to a flying Rydberg atom followed by a projective DV measurement of the atom [28, 40] and through a strong, dispersive interaction with a superconducting transmon qubit 41.

In addition to the coupling of two-level systems to the $\mathrm{CVs}$ of the electro-magnetic field, recently there has also been significant progress in understanding and implementing the coupling of a two-level system to the continuous position and momentum variables of a mechanical oscillator. In a pioneering experiment it was shown that by strongly coupling a superconducting phase qubit to a ground state cooled mechanical oscillator, it was possible to generate a single excitation (single phonon Fock state) of the oscillator [42, see Fig. 11. Various other approaches enabling a strong coupling of a two-level system (e.g. a quantum dot [43, an NV center in diamond [44, an atom 45 and a two-level defect [46]) to a mechanical oscillator have been proposed and some recent preliminary steps have been realized $47+50$. These schemes promise the formation of mechanical oscillators in arbitrary superposition states including the cat state, which in principle can be mapped onto the electromagnetic field 51. This might well be the future route to determinisitic generation of non-Gaussian states for QIP. 
a)
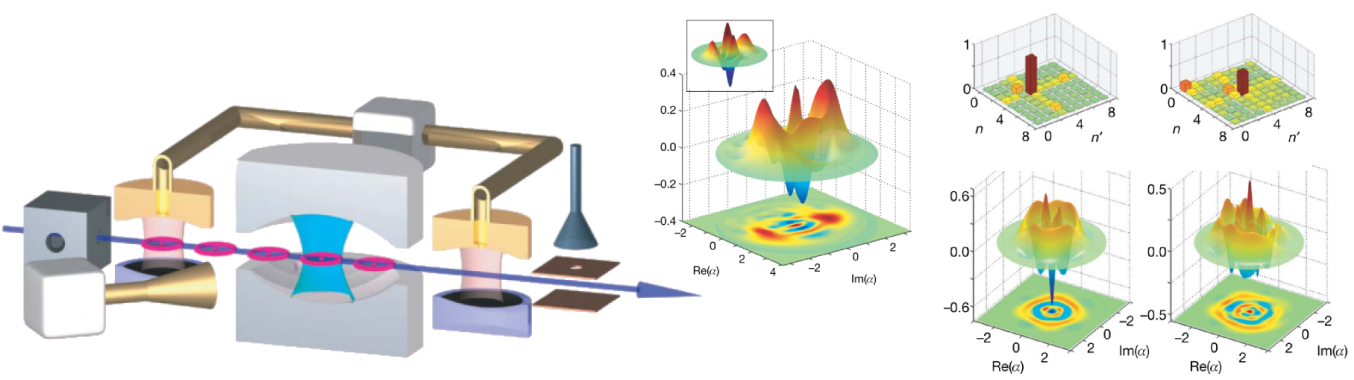

b)

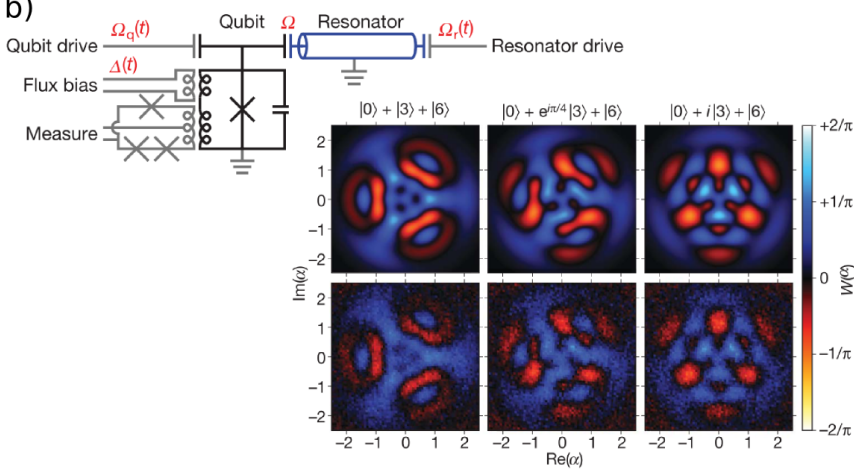

d)
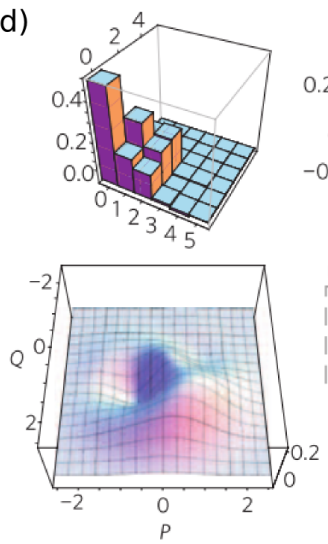

C)

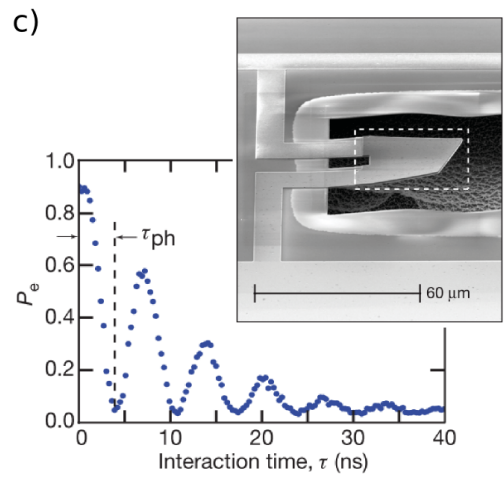

e)
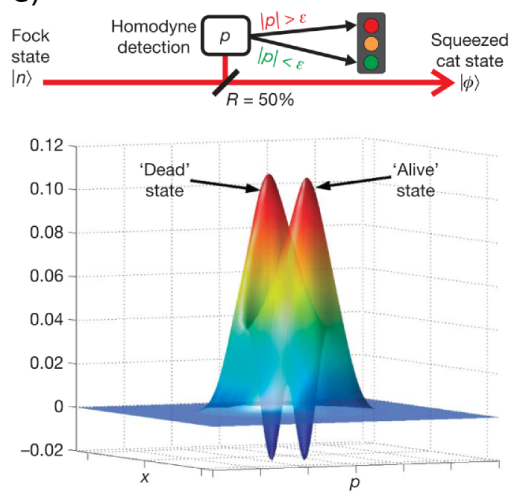

FIG. 1: Examples of non-Gaussian state generation in various systems. a) Schrödinger cat and Fock states of a microwave cavity field induced by the detection of dispersively coupled Rydberg atoms 28, b) arbitrary Fock state superpositions in a waveguide resonator field coupled to a superconducting phase qubit [36], c) population exchange of a single excitation between a superconducting phase qubit and a piezoelectric mechanical oscillator cooled to its ground state 42, d) arbitrary Fock state superpositions of an optical mode through spontaneous parametric down-conversion and coherent-state injected photon detectors [52, e) squeezed Schrödinger cat state of an optical mode induced by conditional homodyne detection on a 2-photon Fock state [53.

Probabilistic generation of non-Gaussian states using finite-level detection systems. In the optical regime, the non-Gaussian transformation is notoriously difficult to implement due to the non-existence of sufficiently strong, optical non-linearities. As an alternative to the deterministic scheme, one may generate the transformation probabilistically using a measurement-induced nonlinearity by applying a non-Gaussian projector. The simplest of these is a single-photon detection, often used for DV QIP.
An essential workhorse for both DV and CV optical QIP is the parametric amplifier (OPA). The OPA operates by the process of spontaneous parametric downconversion where a single pump photon is converted into two lower-frequency photons, called signal and idler, in a nonlinear medium. As the process is spontaneous the probability of generating one or more photon pairs is small, as reflected in the Fock basis representation of the OPA output,

$$
|\phi\rangle \propto\left|0_{s} 0_{i}\right\rangle+\sqrt{\lambda}\left|1_{s} 1_{i}\right\rangle+\lambda\left|2_{s} 2_{i}\right\rangle+\ldots,
$$




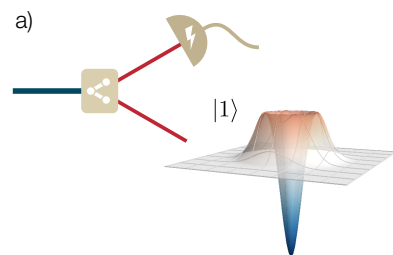

b)

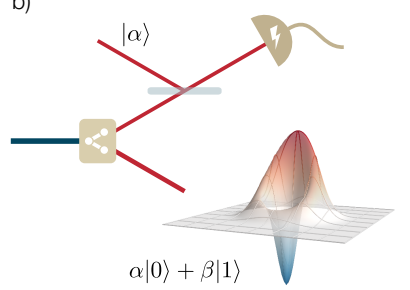

c)

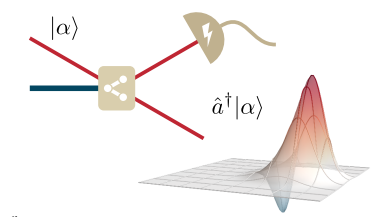

d)

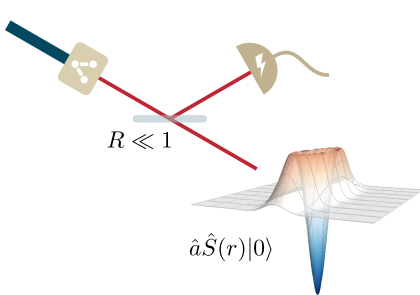

e)

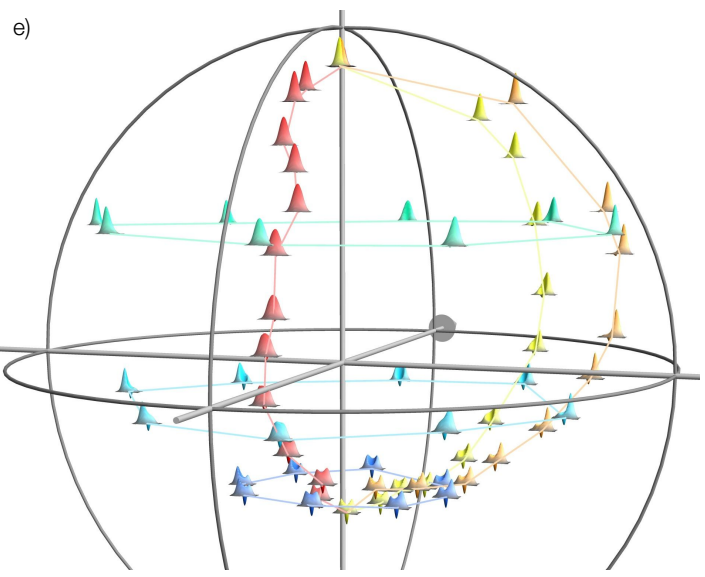

FIG. 2: Examples of heralded non-Gaussian state preparation schemes that have been demonstrated and characterized by quantum homodyne tomography. a) Generation of a single-photon state by SPDC followed by single photon detection b) Engineering of a qubit via displacement and single photon detection c) Addition of a single photon by driven PDC followed by photon detection d) Generation of a kitten state by subtracting a single photon from a squeezed vacuum state e) Wigner functions of various kitten state qubits illustrated on the qubit Bloch sphere 54.

with $\lambda$ related to the gain of the amplifier. It is clear that the signal (s) and idler (i) modes are photon number correlated, but despite of this, the state is Gaussian and thus not directly suitable for various QIP tasks. However, by combining the CV Gaussian entanglement source with a DV detector it is possible to probabilistically induce the generation of a non-Gaussian state. The detection of a photon in the idler mode of a low-gain OPA heralds the existence of its signal twin (see Fig. 2a). The heralding means that this photon can be rigorously characterized by CV homodyne tomography 55 57. By using photonnumber-resolving detection, higher photon number Fock states can be generated 58, 59. The signal mode of the OPA can be seeded with a Gaussian state, like a thermal or coherent beam, as illustrated in Fig. 22. The detection of the idler photon then heralds a photon added version of the seeded state, $\left|\psi_{\text {herald }}\right\rangle \propto \hat{a}^{\dagger}\left|\psi_{\text {seed }}\right\rangle$, which has now turned non-Gaussian 60.

An experimentally easier operation is the reverse process, namely photon subtraction. This can be implemented simply by tapping off a small portion of the beam on a beam splitter with reflectivity $R \ll 1$ and monitoring the reflected part with a photon detector (see Fig. 2d). The detection of a photon heralds the subtraction of a photon from the state in the transmitted beam, $\left|\psi_{\text {out }}\right\rangle \propto \hat{a}\left|\psi_{\text {in }}\right\rangle$ 61. Coherent states are eigenstates of the annihilation operator, so photon subtraction has no effect on coherent and thermal states (which are coherent state mixtures). With other initial states, though, photon subtraction acts as a "de-Gaussifying" operation. Prominently, this has been shown in several experiments with single-mode squeezed vacuum as input (the state in (3) with degenerate $s$ and $i$ modes) with subtraction of a single photon 62 65] and later two or three photons 66 68. The considerable interest in the photon-subtracted squeezed vacuum stems from the recognition 69 that such states are close-to-ideal approximations to a superposition of coherent states (a "Schrödinger's cat") with small amplitudes, also known as a kitten state.

With just a single photon detector to provide a nonGaussian element, CV Gaussian operations like phasespace displacement and homodyne detection can be added to the mix to increase the variety of state generation and manipulation. The displacement operation is easily performed by mixing with a strong coherent state on a highly imbalanced beam splitter. If, as shown in Fig. 2p, a displacement is performed on the idler mode in a heralded single photon setup, one can prepare an arbitrary superposition of the vacuum and the single photon state - a single-rail photonic qubit, $c_{0}|0\rangle+c_{1}|1\rangle$ - with the coefficients controlled by the amplitude and phase of the displacement 7072 . This can of course be extended to higher photon numbers by increasing the number of detectors [52, 73] as in Fig. 11. Similarly, displacement before photon subtraction in a kitten state generation setup allows for arbitrary superpositions of the odd and even states 54. Experimental examples of such superpositions are shown on the Bloch sphere in Fig. 2e. The amount of control that can be achieved by these simple means is such that any single-mode quantum state can be generated by successive applications of phase-space displacement and either photon addition or subtraction [74, 75]. Even more flexibility would be obtained by employing a photon-number resolving detector in combination with the displacement. Such a hybrid detector was characterized by full tomography in Ref. [76. As an alternative to displacement, a homodyne detection on one mode of a two-mode state can also induce arbitrary superposition states like the single-rail qubits conditionally [77. The homodyne measurement outcome then deter- 
mines the coefficients.

By combining these hybrid techniques, the possibilities of quantum state engineering and information processing become countless. Several schemes for increasing the amplitude of kitten states have been proposed, for example by conditional photon counting or homodyne detection on a combination of multiple kittens [78, 80], displacement-improved photon subtraction 73, 81, temporally separated two-photon subtraction 82, or conditional squeezing of a Fock state by homodyne projection [53], see Fig. 11. The energy of one mode of a single-photon entangled state can be increased by displacement [83, 84]. Entangled coherent states can be generated by non-local photon subtraction over a lossy channel 85, and these entangled cat states can be made arbitrarily large through conditional homodyne detection 86. Finally, different ways of inducing hybrid entanglement of cat states or coherent states with a microscopic degree of freedom - a manifestation of Schödinger's cat-atom entanglement - have recently been proposed [87, 88, and experimentally demonstrated [89, 90.

The probabilistic execution of a non-Gaussian transformation of Gaussian states by a non-Gaussian measurement has also been proposed for other systems. This includes the formation of non-Gaussian spin states [91, 92 as well as non-Gaussian states of mechanical oscillators [93, 94 by means of photon counting detections. These schemes are however experimentally challenging and have not yet been realized, although some progress towards a heralded non-Gaussian spin state has been made [91].

\section{QUANTUM INFORMATION PROCESSING}

In the preceding section, we described how hybridization between DV and CV devices and states can be exploited to engineer exotic, non-classical, non-Gaussian quantum states. We shall now discuss possible schemes for quantum information processing that incorporate the above-mentioned hybrid techniques. These include the fundamental tasks of quantum teleportation, quantum error correction (detection), and entanglement distillation, as well as testing Bell inequalities and performing Bell measurements. Ultimately, they would aim at the realization of scalable quantum communication and universal, fault-tolerant quantum computation.

Hybrid quantum teleportation. The most elementary protocol in quantum communication is quantum teleportation [95] - the reliable transfer of arbitrary quantum states using shared entanglement and classical communication; and the most obvious hybrid approach to quantum teleportation is $\mathrm{CV}$ quantum teleportation [96] of DV states or DV quantum teleportation of $\mathrm{CV}$ states. In the optical domain, the former can be, in principle, straightforwardly applied upon any quantum states including single-photon-based qubits. This teleporter possesses the great advantage of being determin-

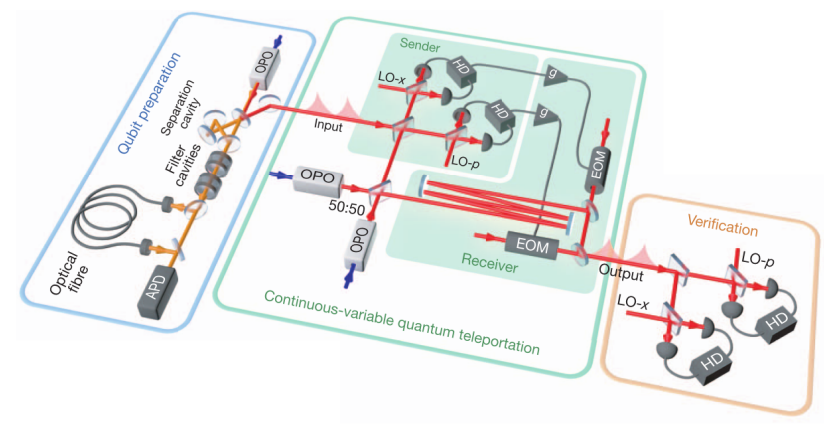

FIG. 3: Schematic layout of CV teleportation of a DV qubit, as implemented in Ref. [97. At left, a time-bin qubit is prepared. The low-gain OPO (optical parametric oscillator) spontaneously emits signal and idler photons which in this case are separated based on their different frequencies. The idler photon traverses an imbalanced Mach-Zender interferometer after which it is observed by a photon detector (APD, avalanche photo diode). Since the two possible paths (short or long) through the interferometer are indistinguishable, the detection of the idler heralds the signal photon (labelled "input") in a qubit state - a superposition of an early $(|0\rangle)$ and a late $(|1\rangle)$ time-bin [99]. In the middle is the CV teleportation device. Mixing the outputs of two high-gain OPOs results in a two-mode squeezed state. One part of this entangled state is mixed with the input qubit followed by a CV Bell measurement consisting of $x / p$ homodyne detectors (HD), while the other part is sent to the receiving station. Here it is modulated with electro-optic modulators (EOM) by the Bell detector outputs amplified with a variable gain. This results - deterministically - in a teleported output qubit state almost identical to the input as verified by the homodyne detection at the right.

istic with solely linear components. However, the price to pay is the intrinsically limited performance: Perfectly faithful and deterministic teleportation of an arbitrary state can only be attained in the limit of an unphysical, infinite degree of Gaussian entanglement. Deterministic $\mathrm{CV}$ teleportation of DV states has recently been demonstrated on photonic qubits 97] and also for a cat state 98. (see Fig. 3).

The converse quantum teleporter, using DV entanglement and DV operations to transfer a CV state, requires breaking up a high-dimensional CV state into states of smaller dimension and performing correspondingly many individual DV teleportations [100 102]. In contrast to the standard CV teleporter, the optical DV teleporter can reach fidelities of $100 \%$. However, its efficiency is fundamentally limited by the probabilistic nature of qubit Bell measurements with linear transformations 103. Only by the use of non-Gaussian transformations or non-Gaussian ancillary states can the teleporter become (near-)deterministic.

Hence, the example of quantum teleportation illustrates nicely what an optical hybrid approach does: it can turn an otherwise probabilistic, linear-optical qubit teleporter into a fully deterministic device, possibly at the expense of the transfer fidelity; and it can make use 
of a potentially high-fidelity transfer of low-dimensional optical states for reliably transmitting a CV state, at the expense of a non-unit success probability. This new level of versatility is, of course, even greater when matter systems are included, as the light-matter interactions offer an alternative way of performing efficient Bell measurements. In fact, using atomic ensembles or two-level emitters, such hybrid light-matter teleportations have been already proposed for long-distance quantum communication 104 and, on a small scale, experimentally demonstrated 105, 106.

Cat-state qubits (defined as $a|\alpha\rangle+b|-\alpha\rangle$ where $a, b$ are complex numbers) may also be teleported using an entangled state of two cat-qubits such as $|\alpha, \alpha\rangle+|-\alpha,-\alpha\rangle$. This protocol has been experimentally demonstrated for binary coherent states [107, and it forms the critical element of cat-qubit quantum computing [108 110] of which a few probabilistic gates have been realised [111, 112]. It is also interesting to note that the efficient distribution of entangled cat states for cat state teleportation can be achieved using a another hybrid approach that combines DV and CV measurements 86.

Hybrid entanglement distillation and quantum communication. For quantum communication based upon the distribution of entangled states, like in a quantum repeater, it is desirable to initially prepare and distribute optical entanglement with high efficiency. Since the CV Gaussian entangled states can be produced in an unconditional fashion, they may serve as a deterministic source of shared entanglement, thus saving expensive storage times in a fully fledged quantum repeater. However, Gaussian entanglement is very sensitive to photon losses and hence entanglement distillation will be absolutely necessary. Solely using CV Gaussian operations does not allow for distilling high-quality Gaussian entanglement from low-quality, noisy Gaussian entanglement $11-13$. The required non-Gaussian element may then be introduced through photon subtraction. For the pure, photon-number correlated, two-mode squeezed state, local photon subtractions acting on the two-mode number states as $(\hat{a} \otimes \hat{a})|n, n\rangle=n|n-1, n-1\rangle$ would smoothen the photon-number distribution, effectively enhancing the entanglement of the state [113. Such an enhancement can also be obtained when the initial states are mixed and noisy after a lossy channel transmission [114, 115]. Experiments were already performed, showing such photon subtraction-based distillation [116, 117].

Apart from photon subtraction, photon addition can be a useful DV operation to locally improve the entanglement of bipartite Gaussian states 118 . In fact, coherent linear combinations of photon subtraction $\propto \hat{a}$ and addition $\propto \hat{a}^{\dagger}$ may be the optimal choice 60] ; and one way to obtain such superpositions is once again a hybrid technique: combine DV photon subtraction with a CV squeezing operation, $\hat{S}^{\dagger}(r) \hat{a} \hat{S}(r)=\cosh r \hat{a}+\sinh r \hat{a}^{\dagger}$ where $r$ is the squeezing parameter. Notice that in this case, squeezing would be promoted from a sole offline resource to an online tool performed locally and indi- vidually on the two halves of a two-mode CV Gaussian state.

As squeezing, in conjunction with photon subtraction, allows for obtaining new kinds of hybrid operations - adding and subtracting light particles in a coherent, wave-like fashion - it opens up completely new possibilities for quantum communication, for instance, by optimizing entanglement distillation schemes [119]. Remarkably, even simpler hybrid operations such as combinations of $\mathrm{CV}$ phase-space displacements with DV photon subtractions may still allow for such improvements 120, 121. The effect of these entanglement-enhancing operations is to non-locally transform the initial CV Gaussian states into, in lowest order, DV qubit-type entangled states - a kind of bipartite hybrid state engineering at a distance.

Besides supplementing photon subtraction by squeezing or displacement operations, there are other recently proposed techniques that allow for optically realizing entanglement distillation. One such prominent method is the heralded, noiseless linear amplifier (NLA) introduced by Lund and Ralph [122] and realized in different ways [123 125]. On the operational, application-oriented side, the NLA provides a DV, non-Gaussian tool to distill CV 122 as well as DV 126 entangled states, where in the latter case, this may immediately render atomicensemble-based quantum repeaters 104 more efficient 127. Moreover, the DV NLA can be used to correct errors in $\mathrm{CV}$ teleportation resulting from a lossy distribution of entangled states 128 . On the more conceptual side, the NLA serves as yet another illustrative example for the benefit of a hybrid approach: while the original, non-heralded and deterministic, quantum-limited (phase-insensitive) linear amplifier by Caves [129] is a CV Gaussian operation, only its generalization to the nonGaussian and heralded DV regime enables one to beat supposedly fundamental quantum noise limits, again at the expense of a non-unit success probability.

Hybrid Bell tests and measurements. The list of both fundamental and application-oriented quantum tasks and protocols in a quantum communication setting, i.e., with at least two spatially separated parties sharing classical and quantum resources, can be further extended. There are the fundamental Bell tests, which are to negate local realism even by means of realistic, imperfect detectors, and for this, combined DV and CV measurements may help [130, 131]. Gaussian CV entangled states, having an immediate hidden-variable description in form of a positive Wigner function, can never violate a Bell inequality based upon CV homodyne measurements; only the detection of a set of discrete variables such as photon-number parities 132 would work. In a quantum repeater chain based upon elementary, polarization-entangled photon pairs, the necessary twoqubit Bell measurements for connecting the individual repeater segments can be made more efficient using - once again - local squeezers, in addition to counting photon numbers 133 . 


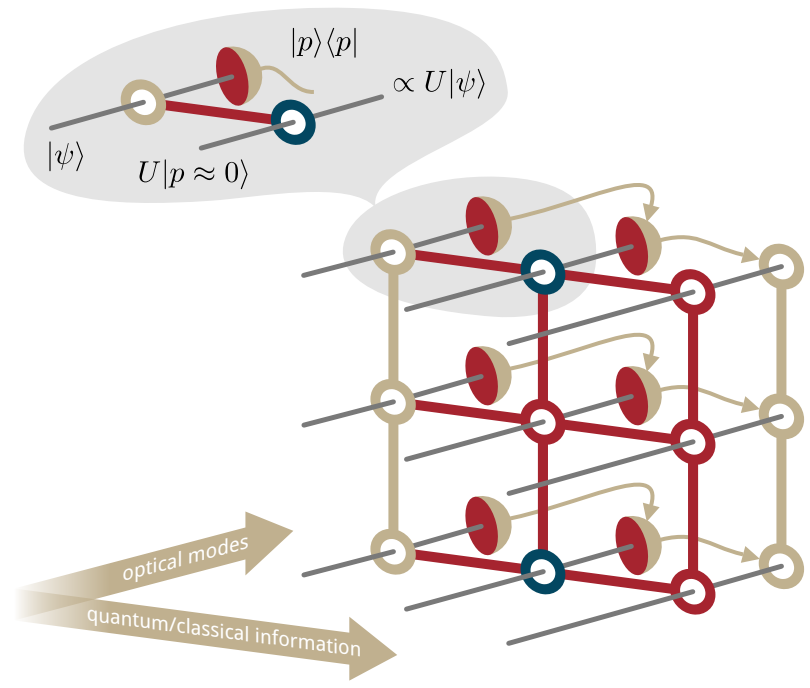

Hybrid quantum computing. While a full-scale quantum repeater can be thought of as a nonlocal device with local, small-scale quantum computers that benefits from the hybrid approach for quantum error detection and entanglement distillation, a large-scale, universal quantum computer would have to combine the universal processing of some logical quantum state with a sufficient degree of fault tolerance in order to maintain an arbitrarily long computation. Examples of universal gate sets leading to universal computation for DV and CV logical encoding are given in BOX 1 and 2.

The optical hybrid approach to implementing universal quantum gates [3, 134 relies on teleportation-based, or, more generally, measurement-based quantum processing [135], similar to Ref. 4]. However, the crucial and with regards to implementations useful difference is that some parts of an otherwise fully DV (CV) measurement-based scheme are replaced by CV (DV) elements.

Consider the elementary circuits in the zoomed grey circles of Fig. 4. an arbitrary input quantum state $|\psi\rangle$, representing one propagating optical mode (indicated by the corresponding thin grey line), gets entangled with either a Gaussian, highly squeezed single-mode state $|p \approx 0\rangle$ (indicated by a red circle) or, alternatively, with a suitable non-Gaussian single-mode state $U|p \approx 0\rangle$ (indicated by a blue circle). The entangling operation is Gaussian in either case (indicated by the thick red line). A universally transformed single-mode output state, $U|\psi\rangle$, can then be produced, either through simple Gaussian homodyne detection projecting the input mode onto the $|p\rangle$ basis (indicated by a red detector) or via a, in general, non-Gaussian projection measurement onto a suitably rotated measurement basis, $U|p\rangle$ (indicated by a blue detector). These elementary processes are reminiscent of CV quantum teleportation, where indeed the final, desired output state would be obtained only depending on some phase-space displacement given by the outcome of the measurement. The application of an arbitrary single-mode gate $U$ may require more than just one such elementary step, where generally every step includes adjusting the measurement basis conditioned upon the previous outcomes and depending on some additional corrections beyond simple displacements. The crucial difference compared with standard quantum teleportation is (besides the measurement being only single-mode instead of a two-mode Bell measurement) that an input state is not only transferred, but also manipulated.

Using these elementary schemes, one may realize two important gates of the CV universal gate set: a universal squeezer [136] (experimentally applied to a single photon in Ref. [137]) and a cubic phase gate [138. For the case, when the ancilla state is non-Gaussian $(U|p \approx 0\rangle)$ and the desired gate is weak and, for instance, cubic, a suitable three-photon superposition state (as demonstrated in Ref. 73] ) may be employed [139].

In order to achieve universal processing of arbitrary multi-mode quantum states, the elementary teleportation circuits can be concatenated in a so-called cluster- 
state computation [135, 140. The input state is then teleported into (or prepared within) a sufficiently large, multi-mode, non-Gaussian (or Gaussian) cluster state, which is then subject to CV Gaussian (or arbitrary, including DV non-Gaussian) measurements (see Fig. 47. While universality does not depend on whether the nonGaussian elements appear in the detectors or in the ancilla states, the choice of hybridization will often be based on the actual complexity of the implementation.

Similar to quantum communication, hybrid schemes for quantum computation also greatly benefit from combining light and matter systems. Again, the additional possibility of coupling light with matter, that is more specifically, CV oscillators with DV two-level systems, extends the toolbox of gate operations to include highly nonlinear interactions. This allows for deterministic universal gate operations on the qubits [141, where again the light mode acts as a quantum bus that mediates the qubit gates. However, this time the light mode is no longer measured out, but instead it disentangles automatically from the qubit systems when a full sequence of light-matter interactions has been completed. Suitable interactions for this purpose are the controlled phase rotations induced by dispersive, cavity QED-type atomlight interactions [142] or the naturally occurring, controlled phase-space displacements of a microwave mode depending on the discrete state of a superconducting qubit [143. It is important to notice that in these schemes, in principle, weak nonlinear interactions are sufficient, which result in small phase shifts or displacements that can be effectively enhanced by increasing the amplitude of the CV quantum bus.

\section{OUTLOOK}

Less than a decade ago, the boundary between DV and $\mathrm{CV}$ platforms for quantum information processing was extremely sharp. This is no longer the case. The boundary is progressively becoming smeared out as a result of several recent advances in combining the technologies of the two platforms. This marriage of the two different disciplines, as reviewed in this article, has led to a wealth of new theoretical proposals and some experimental implementations of new promising protocols for quantum information processing. However, the field is still very young and researchers might only have scratched the surface of a much larger and richer field.

Most of the demonstrations to date are proof-ofprinciple experiments with a lack of high-fidelity operation, efficiency and scalability. To advance the field in the direction of higher fidelity operation and higher efficiency, a deeper understanding of the present limitations must be attained in order to devise new systems with fidelities and efficiencies that are sufficiently high to allow for perfect error correction and thus fault tolerant QIP. To facilitate scalability, the systems must be miniaturized: E.g. using integrated photonics, phononics and electronics for squeezed light generation and propagation; solid state platforms containing finite-level systems for single photon generation and non-Gaussian transformations; and integrated on-chip detector technology for efficient detection of either CVs and DVs combined with real-time feedback.
[1] Nielsen, M. A. \& Chuang, I. L. Quantum computation and quantum information (Cambridge University Press, 2000).

[2] Ladd, T. D. et al. Quantum computers. Nature 464, 45-53 (2010).

[3] van Loock, P. Optical hybrid approaches to quantum information. Laser $\&$ Photonics Reviews 5, 167-200 (2011).

[4] Knill, E., Laflamme, R. \& Milburn, G. J. A scheme for efficient quantum computation with linear optics. Nature 409, 46-52 (2001).

[5] Kok, P., Nemoto, K., Ralph, T. C., Dowling, J. P. \& Milburn, G. J. Linear optical quantum computing with photonic qubits. Reviews of Modern Physics 79, 135174 (2007).

[6] Furusawa, A. et al. Unconditional Quantum Teleportation. Science 282, 706-709 (1998).

[7] Grosshans, F. et al. Quantum key distribution using gaussian-modulated coherent states. Nature 421, 23841 (2003).

[8] Aoki, T. et al. Quantum error correction beyond qubits. Nature Physics 5, 541-546 (2009).

[9] Lassen, M. et al. Quantum optical coherence can survive photon losses using a continuous-variable quantum erasure-correcting code. Nature Photonics 4, 700-705 (2010).

[10] Bartlett, S. D., Sanders, B. C., Braunstein, S. L. \& Nemoto, K. Efficient classical simulation of continuous variable quantum information processes. Physical Review Letters 88, 097904 (2002).

[11] Fiurášek, J. Gaussian transformations and distillation of entangled Gaussian states. Physical Review Letters 89, 137904 (2002).

[12] Eisert, J., Scheel, S. \& Plenio, M. B. Distilling Gaussian states with gaussian operations is impossible. Physical Review Letters 89, 137903 (2002).

[13] Giedke, G. \& Ignacio Cirac, J. Characterization of Gaussian operations and distillation of Gaussian states. Physical Review A 66, 032316 (2002).

[14] Niset, J., Fiurášek, J. \& Cerf, N. J. No-go theorem for Gaussian quantum error correction. Physical Review Letters 102, 120501 (2009).

[15] Braunstein, S. L. \& van Loock, P. Quantum information with continuous variables. Reviews of Modern Physics 77, 513-577 (2005).

[16] Andersen, U. L., Leuchs, G. \& Silberhorn, C. Continuous-variable quantum information processing. Laser $\backslash$ E Photonics Reviews 4, 337-354 (2010). 
[17] Weedbrook, C. et al. Gaussian quantum information. Reviews of Modern Physics 84, 621-669 (2012).

[18] Castellanos-Beltran, M. A., Irwin, K. D., Hilton, G. C., Vale, L. R. \& Lehnert, K. W. Amplification and squeezing of quantum noise with a tunable Josephson metamaterial. Nature Physics 4, 929-931 (2008).

[19] Bergeal, N., Schackert, F., Frunzio, L. \& Devoret, M. Two-mode correlation of microwave quantum noise generated by parametric down-conversion. Physical review letters (2012).

[20] Eichler, C. et al. Observation of Two-Mode Squeezing in the Microwave Frequency Domain. Physical Review Letters 107, 113601 (2011).

[21] Eichler, C., Bozyigit, D. \& Wallraff, a. Characterizing quantum microwave radiation and its entanglement with superconducting qubits using linear detectors. Physical Review A 86, 032106 (2012).

[22] Estève, J., Gross, C., Weller, a., Giovanazzi, S. \& Oberthaler, M. K. Squeezing and entanglement in a Bose-Einstein condensate. Nature 455, 1216-1219 (2008).

[23] Rudner, M. S., Vandersypen, L. M. K., Vuletić, V. \& Levitov, L. S. Generating Entanglement and Squeezed States of Nuclear Spins in Quantum Dots. Physical Review Letters 107, 206806 (2011).

[24] Bennett, S. et al. Phonon-Induced Spin-Spin Interactions in Diamond Nanostructures: Application to Spin Squeezing. Physical Review Letters 110, 156402 (2013).

[25] Aspelmeyer, M., Kippenberg, T. J. \& Marquardt, F. Cavity Optomechanics. arXiv 1303.0733 (2013).

[26] Palomaki, T. a., Teufel, J. D., Simmonds, R. W. \& Lehnert, K. W. Entangling mechanical motion with microwave fields. Science (New York, N.Y.) 342, 710-3 (2013).

[27] Eisaman, M. D., Fan, J., Migdall, A. \& Polyakov, S. V. Invited Review Article: Single-photon sources and detectors. Review of Scientific Instruments 82, 071101 (2011).

[28] Deléglise, S. et al. Reconstruction of non-classical cavity field states with snapshots of their decoherence. Nature 455, 510-4 (2008).

[29] Eichler, C. et al. Experimental State Tomography of Itinerant Single Microwave Photons. Physical Review Letters 106, 220503 (2011).

[30] Mallet, F. et al. Quantum State Tomography of an Itinerant Squeezed Microwave Field. Physical Review Letters 106, 220502 (2011).

[31] Menzel, E. P. et al. Dual-Path State Reconstruction Scheme for Propagating Quantum Microwaves and Detector Noise Tomography. Physical Review Letters 105, 100401 (2010).

[32] MacRae, A., Brannan, T., Achal, R. \& Lvovsky, A. I. Tomography of a high-purity narrowband photon from a transient atomic collective excitation. Physical Review Letters 109, 8 (2011).

[33] Bimbard, E. et al. Homodyne Tomography of a Single Photon Retrieved on Demand from a Cavity-Enhanced Cold Atom Memory. Physical Review Letters 112, 033601 (2014).

[34] Law, C. K. \& Eberly, J. H. Arbitrary control of a quantum electromagnetic field. Physical Review Letters 76, 1055-1058 (1996).

[35] Hofheinz, M. et al. Generation of Fock states in a superconducting quantum circuit. Nature 454, 310-4 (2008).
[36] Hofheinz, M. et al. Synthesizing arbitrary quantum states in a superconducting resonator. Nature 459, 5469 (2009).

[37] Yurke, B. \& Stoler, D. Generating quantum mechanical superpositions of macroscopically distinguishable states via amplitude dispersion. Physical Review Letters 57, 13-16 (1986).

[38] Schrödinger, E. Die gegenwärtige Situation in der Quantenmechanik. Die Naturwissenschaften 23, 807-812 (1935).

[39] Monroe, C., Meekhof, D. M., King, B. E. \& Wineland, D. J. A "Schrödinger cat" superposition state of an atom. Science 272, 1131-1136 (1996).

[40] Brune, M. et al. Observing the progressive decoherence of the "meter" in a quantum measurement. Physical review letters $\mathbf{7 7}, 4887-4890$ (1996).

[41] Vlastakis, B. et al. Deterministically Encoding Quantum Information Using 100-Photon Schrodinger Cat States. Science (New York, N.Y.) 607 (2013).

[42] O'Connell, A. D. et al. Quantum ground state and single-phonon control of a mechanical resonator. $\mathrm{Na}$ ture 464, 697-703 (2010).

[43] Wilson-Rae, I., Zoller, P. \& Imamolu, a. Laser Cooling of a Nanomechanical Resonator Mode to its Quantum Ground State. Physical Review Letters 92, 075507 (2004).

[44] Rabl, P. et al. Strong magnetic coupling between an electronic spin qubit and a mechanical resonator. Physical Review B 79, 041302 (2009).

[45] Hammerer, K. et al. Strong Coupling of a Mechanical Oscillator and a Single Atom. Physical Review Letters 103, 063005 (2009).

[46] Ramos, T., Sudhir, V., Stannigel, K., Zoller, P. \& Kippenberg, T. J. Nonlinear Quantum Optomechanics via Individual Intrinsic Two-Level Defects. Physical Review Letters 110, 193602 (2013).

[47] Bennett, S. D., Cockins, L., Miyahara, Y., Grütter, P. \& Clerk, A. a. Strong Electromechanical Coupling of an Atomic Force Microscope Cantilever to a Quantum Dot. Physical Review Letters 104, 017203 (2010).

[48] Yeo, I. et al. Strain-mediated coupling in a quantum dot-mechanical oscillator hybrid system. Nature Nanotechnology 9, 106-10 (2014).

[49] Arcizet, O. et al. A single nitrogen-vacancy defect coupled to a nanomechanical oscillator. Nature Physics $\mathbf{7}$, 879-883 (2011).

[50] Kolkowitz, S. et al. Coherent sensing of a mechanical resonator with a single-spin qubit. Science $\mathbf{3 3 5}, 1603-6$ (2012).

[51] Stannigel, K., Rabl, P., Sø rensen, A. S., Zoller, P. \& Lukin, M. D. Optomechanical Transducers for LongDistance Quantum Communication. Physical Review Letters 105, 220501 (2010).

[52] Bimbard, E., Jain, N., MacRae, A. \& Lvovsky, A. I. Quantum-optical state engineering up to the twophoton level. Nature Photonics 4, 243-247 (2010).

[53] Ourjoumtsev, A., Jeong, H., Tualle-Brouri, R. \& Grangier, P. Generation of optical 'Schrödinger cats' from photon number states. Nature 448, 784-6 (2007).

[54] Neergaard-Nielsen, J. S. et al. Optical continuousvariable qubit. Physical Review Letters 105, 053602 (2010).

[55] Lvovsky, A. I. et al. Quantum state reconstruction of the single-photon Fock state. Physical Review Letters 
87, 050402 (2001).

[56] Neergaard-Nielsen, J. S., Nielsen, B. M., Takahashi, H., Vistnes, A. I. \& Polzik, E. S. High purity bright single photon source. Optics Express 15, 7940-7949 (2007).

[57] Morin, O., DAuria, V., Fabre, C. \& Laurat, J. Highfidelity single-photon source based on a Type II optical parametric oscillator. Optics Letters 37, 3738 (2012).

[58] Ourjoumtsev, A., Tualle-Brouri, R. \& Grangier, P. Quantum homodyne tomography of a two-photon Fock state. Physical Review Letters 96, 213601 (2006).

[59] Cooper, M., Wright, L., Söller, C. \& Smith, B. Experimental generation of multi-photon Fock states. Optics Express 21, 5311-5317 (2013).

[60] Zavatta, A., Viciani, S. \& Bellini, M. Quantum-toclassical transition with single-photon-added coherent states of light. Science 306, 660-662 (2004).

[61] Ban, M. Quasicontinuous measurements of photon number. Physical Review A 49, 5078-5085 (1994).

[62] Wenger, J., Tualle-Brouri, R. \& Grangier, P. NonGaussian statistics from individual pulses of squeezed light. Physical Review Letters 92, 153601 (2004).

[63] Ourjoumtsev, A., Tualle-brouri, R., Laurat, J. \& Grangier, P. Generating optical Schrödinger kittens for quantum information processing. Science 312, 83-86 (2006).

[64] Neergaard-Nielsen, J. S., Nielsen, B. M., Hettich, C., Mø lmer, K. \& Polzik, E. S. Generation of a superposition of odd photon number states for quantum information networks. Physical Review Letters 97, 083604 (2006).

[65] Wakui, K., Takahashi, H., Furusawa, A. \& Sasaki, M. Photon subtracted squeezed states generated with periodically poled KTiOPO 4. Optics Express 15, 35683574 (2007).

[66] Takahashi, H. et al. Generation of large-amplitude coherent-state superposition via ancilla-assisted photon subtraction. Physical Review Letters 101, 233605 (2008).

[67] Gerrits, T. et al. Generation of optical coherent-state superpositions by number-resolved photon subtraction from the squeezed vacuum. Physical Review A 82, 031802(R) (2010).

[68] Namekata, N. et al. Non-Gaussian operation based on photon subtraction using a photon-number-resolving detector at a telecommunications wavelength. Nature Photonics 4, 655-660 (2010).

[69] Dakna, M., Anhut, T., Opatrný, T., Knöll, L. \& Welsch, D.-G. Generating Schrödinger-cat-like states by means of conditional measurements on a beam splitter. Physical Review A 55, 3184-3194 (1997).

[70] Lvovsky, A. I. \& Mlynek, J. Quantum-optical catalysis: Generating nonclassical states of light by means of linear optics. Physical Review Letters 88, 250401 (2002).

[71] Resch, K., Lundeen, J. S. \& Steinberg, A. Quantum state preparation and conditional coherence. Physical Review Letters 88, 113601 (2002).

[72] Babichev, S. A., Ries, J. \& Lvovsky, A. I. Quantum scissors: Teleportation of single-mode optical states by means of a nonlocal single photon. Europhysics Letters 64, 1-7 (2003).

[73] Yukawa, M. et al. Generating superposition of up-to three photons for continuous variable quantum information processing. Optics Express 21, 5 (2013).

[74] Dakna, M., Clausen, J., Knöll, L. \& Welsch, D. D.-G. D.-G. D.-G. Generation of arbitrary quantum states of traveling fields. Physical Review A 59, 1658-1661 (1999).

[75] Fiurášek, J., García-Patrón, R. \& Cerf, N. Conditional generation of arbitrary single-mode quantum states of light by repeated photon subtractions. Physical Review A 72, 033822 (2005).

[76] Zhang, L. et al. Mapping coherence in measurement via full quantum tomography of a hybrid optical detector. Nature Photonics 6, 364-368 (2012).

[77] Babichev, S. A., Brezger, B. \& Lvovsky, A. I. Remote preparation of a single-mode photonic qubit by measuring field quadrature noise. Physical Review Letters 92, 047903 (2004).

[78] Lund, A. P., Jeong, H., Ralph, T. C. \& Kim, M. Conditional production of superpositions of coherent states with inefficient photon detection. Physical Review A 70, 020101 (2004).

[79] Takeoka, M. \& Sasaki, M. Conditional generation of an arbitrary superposition of coherent states. Physical Review A 75, 064302 (2007).

[80] Laghaout, A. et al. Amplification of realistic Schrödinger-cat-state-like states by homodyne heralding. Physical Review A 87, 043826 (2013).

[81] Nielsen, A. \& Mø lmer, K. Transforming squeezed light into a large-amplitude coherent-state superposition. Physical Review A 76, 043840 (2007).

[82] Takeoka, M., Takahashi, H. \& Sasaki, M. Largeamplitude coherent-state superposition generated by a time-separated two-photon subtraction from a continuous-wave squeezed vacuum. Physical Review A 77, 062315 (2008).

[83] Bruno, N. et al. Displacement of entanglement back and forth between the micro and macro domains. Nature Physics 9, 545-548 (2013).

[84] Lvovsky, A. I., Ghobadi, R., Chandra, A., Prasad, a. S. \& Simon, C. Observation of micromacro entanglement of light. Nature Physics 9, 541-544 (2013).

[85] Ourjoumtsev, A., Ferreyrol, F., Tualle-Brouri, R. \& Grangier, P. Preparation of non-local superpositions of quasi-classical light states. Nature Physics 5, 189-192 (2009).

[86] Brask, J. B., Rigas, I., Polzik, E., Andersen, U. L. \& Sø rensen, A. S. Hybrid long-distance entanglement distribution p rotocol. Physical Review Letters 105, 160501 (2010).

[87] Ghobadi, R., Lvovsky, A. I. \& Simon, C. Creating and Detecting Micro-Macro Photon-Number Entanglement by Amplifying and Deamplifying a Single-Photon Entangled State. Physical Review Letters 110, 170406 (2013).

[88] Andersen, U. L. \& Neergaard-Nielsen, J. S. Heralded generation of a micro-macro entangled state. Physical Review A 88, 022337 (2013).

[89] Jeong, H. et al. Generation of hybrid entanglement of light. Nature Photonics 8, 564-569 (2014).

[90] Morin, O. et al. Remote creation of hybrid entanglement between particle-like and wave-like optical qubits. Nature Photonics 8, 570-574 (2014).

[91] Christensen, S. L. et al. Toward quantum state tomography of a single polariton state of an atomic ensemble. New Journal of Physics 15, 015002 (2013).

[92] McConnell, R. et al. Generating entangled spin states for quantum metrology by single-photon detection. Physical Review A 88, 063802 (2013). 
[93] Paternostro, M. Engineering Nonclassicality in a Mechanical System through Photon Subtraction. Physical Review Letters 106, 183601 (2011).

[94] Galland, C., Sangouard, N., Piro, N., Gisin, N. \& Kippenberg, T. J. Heralded Single-Phonon Preparation, Storage, and Readout in Cavity Optomechanics. Physical Review Letters 112, 143602 (2014).

[95] Bennett, C. H. et al. Teleporting an unknown quantum state via dual classical and Einstein-Podolsky-Rosen channels. Physical Review Letters 70, 1895-1899 (1993).

[96] Braunstein, S. L. \& Kimble, H. J. Teleportation of Continuous Quantum Variables. Physical Review Letters 80, 869-872 (1998).

[97] Takeda, S., Mizuta, T., Fuwa, M., van Loock, P. \& Furusawa, A. Deterministic quantum teleportation of photonic quantum bits by a hybrid technique. Nature $\mathbf{5 0 0}$, $315-8$ (2013).

[98] Lee, N. et al. Teleportation of nonclassical wave packets of light. Science 332, 330-333 (2011).

[99] Zavatta, A., DAngelo, M., Parigi, V. \& Bellini, M. Remote Preparation of Arbitrary Time-Encoded SinglePhoton Ebits. Physical Review Letters 96, 020502 (2006).

[100] Andersen, U. L. \& Ralph, T. C. High-Fidelity Teleportation of Continuous-Variable Quantum States Using Delocalized Single Photons. Physical Review Letters 111, 050504 (2013).

[101] Marshall, K. \& James, D. F. V. High-fidelity teleportation of continuous-variable quantum states with discrete-variable resources. Journal of the Optical Society of America B 31, 423 (2014).

[102] Kogias, I., Ragy, S. \& Adesso, G. Continuous-variable versus hybrid schemes for quantum teleportation of Gaussian states. Physical Review A 89, 052324 (2014).

[103] Calsamiglia, J. \& Lütkenhaus, N. Maximum efficiency of a linear-optical Bell-state analyzer. Applied Physics $B$ 72, 67-71 (2001).

[104] Duan, L.-M., Lukin, M. D., Cirac, J. I. \& Zoller, P. Long-distance quantum communication with atomic ensembles and linear optics. Nature 414, 413-418 (2001).

[105] Sherson, J. F. et al. Quantum teleportation between light and matter. Nature 443, 557-560 (2006).

[106] Yuan, Z.-S. et al. Experimental demonstration of a BDCZ quantum repeater node. Nature 454, 1098-101 (2008).

[107] Neergaard-Nielsen, J. S., Eto, Y., Lee, C.-w., Jeong, H. \& Sasaki, M. Quantum tele-amplification with a continuous-variable superposition state. Nature Photonics 7, 439-443 (2013).

[108] Jeong, H. \& Kim, M. Efficient quantum computation using coherent states. Physical Review A 65, 042305 (2002).

[109] Ralph, T. C., Gilchrist, A., Milburn, G., Munro, W. J. \& Glancy, S. Quantum computation with optical coherent states. Physical Review A 68, 042319 (2003).

[110] Lund, A. P., Ralph, T. C. \& Haselgrove, H. L. Faulttolerant linear optical quantum computing with smallamplitude coherent States. Physical Review Letters 100, 030503 (2008).

[111] Tipsmark, A. et al. Experimental demonstration of a Hadamard gate for coherent state qubits. Physical Review A 84, 050301(R) (2011).

[112] Blandino, R., Ferreyrol, F., Barbieri, M., Grangier, P. \& Tualle-Brouri, R. Characterization of a $\pi$-phase shift quantum gate for coherent-state qubits. New Journal of Physics 14, 013017 (2012).

[113] Opatrný, T., Kurizki, G. \& Welsch, D.-G. Improvement on teleportation of continuous variables by photon subtraction via conditional measurement. Physical Review A 61, 032302 (2000).

[114] Kitagawa, A., Takeoka, M. \& Sasaki, M. Entanglement evaluation of non-Gaussian states generated by photon subtraction from squeezed states. Physical Review $A$ 73, 042310 (2006).

[115] Zhang, S. L. \& van Loock, P. Distillation of mixedstate continuous-variable entanglement by photon subtraction. Physical Review A 82, 062316 (2010).

[116] Ourjoumtsev, A., Dantan, A., Tualle-Brouri, R. \& Grangier, P. Increasing entanglement between Gaussian states by coherent photon subtraction. Physical Review Letters 98, 030502 (2007).

[117] Takahashi, H. et al. Entanglement distillation from Gaussian input states. Nature Photonics 4, 178-181 (2010).

[118] Navarrete-Benlloch, C., García-Patrón, R., Shapiro, J. \& Cerf, N. Enhancing quantum entanglement by photon addition and subtraction. Physical Review A 86, 012328 (2012).

[119] Zhang, S. \& van Loock, P. Local Gaussian operations can enhance continuous-variable entanglement distillation. Physical Review A 84, 062309 (2011).

[120] Fiurášek, J. Improving entanglement concentration of Gaussian states by local displacements. Physical Review A 84, 012335 (2011).

[121] Tipsmark, A., Neergaard-Nielsen, J. S. \& Andersen, U. L. Displacement-enhanced entanglement distillation of single-mode-squeezed entangled states. Optics Express 21, 6670 (2013).

[122] Ralph, T. C. \& Lund, A. P. Nondeterministic Noiseless Linear Amplification of Quantum Systems. In Lvovsky, A. (ed.) AIP Conference Proceedings, 155-160 (AIP, New York, 2009). URL http://link.aip.org/link/ APCPCS/v1110/i1/p155/s1\&Agg=doi.

[123] Xiang, G. Y., Ralph, T. C., Lund, A. P., Walk, N. \& Pryde, G. J. Heralded noiseless linear amplification and distillation of entanglement. Nature Photonics 4, 316319 (2010).

[124] Ferreyrol, F. et al. Implementation of a nondeterministic optical noiseless amplifier. Physical Review Letters 104, 123603 (2010).

[125] Zavatta, A., Fiurášek, J. \& Bellini, M. A high-fidelity noiseless amplifier for quantum light states. Nature Photonics 5, 52-60 (2010).

[126] Miná, J., de Riedmatten, H. \& Sangouard, N. Quantum repeaters based on heralded qubit amplifiers. Physical Review A 85, 032313 (2012).

[127] Sangouard, N., Simon, C., de Riedmatten, H. \& Gisin, N. Quantum repeaters based on atomic ensembles and linear optics. Reviews of Modern Physics 83, 33-80 (2011).

[128] Ralph, T. C. \& Pryde, G. J. Optical Quantum Computation. Progress in Optics 54, 209-269 (2010).

[129] Caves, C. Quantum-mechanical noise in an interferometer. Physical Review D 23, 1693-1708 (1981).

[130] Cavalcanti, D., Brunner, N., Skrzypczyk, P., Salles, A. \& Scarani, V. Large violation of Bell inequalities using both particle andwave measurements. Physical Review A 84, 022105 (2011). 
[131] Laghaout, A., Björk, G. \& Andersen, U. L. Realistic limits on the nonlocality of an N-partite single-photon superposition. Physical Review A 84, 062127 (2011).

[132] Banaszek, K. \& Wódkiewicz, K. Nonlocality of the Einstein-Podolsky-Rosen state in the Wigner representation. Physical Review A 58, 4345-4347 (1998).

[133] Zaidi, H. a. \& van Loock, P. Beating the One-Half Limit of Ancilla-Free Linear Optics Bell Measurements. Physical Review Letters 110, 260501 (2013).

[134] Furusawa, A. \& van Loock, P. Quantum Teleportation and Entanglement: A Hybrid Approach to Optical Quantum Information Processing (Wiley VCH, Berlin, 2011).

[135] Raussendorf, R. \& Briegel, H. J. A One-Way Quantum Computer. Physical Review Letters 86, 5188-5191 (2001).

[136] Filip, R., Marek, P. \& Andersen, U. L. Measurementinduced continuous-variable quantum interactions. Physical Review A 71, 042308 (2005).

[137] Miwa, Y. et al. Exploring a New Regime for Process- ing Optical Qubits: Squeezing and Unsqueezing Single Photons. Physical Review Letters 113, 013601 (2014).

[138] Gottesman, D., Kitaev, A. \& Preskill, J. Encoding a qubit in an oscillator. Physical Review A 64, 012310 (2001).

[139] Marek, P., Filip, R. \& Furusawa, A. Deterministic implementation of weak quantum cubic nonlinearity. Physical Review A 84, 053802 (2011).

[140] Menicucci, N. et al. Universal Quantum Computation with Continuous-Variable Cluster States. Physical Review Letters 97, 110501 (2006).

[141] Wang, X. \& Zanardi, P. Simulation of many-body interactions by conditional geometric phases. Physical Review A 65, 032327 (2002).

[142] van Loock, P. et al. Hybrid quantum computation in quantum optics. Physical Review A 78, 022303 (2008).

[143] Spiller, T. P. et al. Quantum computation by communication. New Journal of Physics 8, 30-30 (2006). 